\title{
INTERPRETING THE MAIN HI AND CO $\ell-V$ FEATURES IN THE GALACTIC BAR FROM SELF-CONSISTENT STELLAR AND GAS DYNAMICAL MODELS
}

\author{
R. FUX
}

Geneva Observatory, CH-1290 Sauverny, Switzerland

We propose a new picture accounting for the dominant features in the observed $\ell-V$ distribution of the Milky Way gas within the central few kpcs, based on the gas flow in two self-consistent and symmetry-free barred dynamical models. These models are snapshots selected from high resolution 3D $N$-body and SPH simulations with $4 \times 10^{6}$ stellar and dark particles and $1.5 \times 10^{5}$ gas particles, and with initial conditions adapted from Fux (1997). The main results are shown in figure 1 . In both models, the inclination angle of the bar relative to the Sun-Galactic centre line is $25^{\circ}$, the corotation radius close to $4.4 \mathrm{kpc}$ and the sound speed of the gas $10 \mathrm{~km} \mathrm{~s}^{-1}$.

Gas dynamics in a rotating bar is driven by shocks which are believed to generate the dustlanes observed in external barred galaxies (Athanassoula 1992). Such shocks are also expected to exist in the Galaxy and the compressed gas behind them should produce a typical trace in the $\mathrm{HI}$ and $\mathrm{CO}$ $\ell-V$ diagrams. According to our models, the connecting arm is the signature of the near side branch of these dustlane shocks. The shocked gas plunges onto the nuclear disc/ring and is responsible for the terminal velocity peak. The far side branch of the shocks appears as an almost vertical feature in the model $\ell-V$ diagram, which is indeed visible in the CO data near $\ell=-4^{\circ}$. Some other vertical CO features, like the one at $\ell \approx 5.5^{\circ}$, are understood as molecular clouds crossing the shock front and therefore undergoing a strong velocity gradient.

The $135-\mathrm{km} / \mathrm{s}$ arm, long ago suspected as the far side counterpart of the 3 -kpc arm, crosses the $\ell=0$ axis at higher absolute velocity than the latter, because the gas, moving almost parallel to the arm, falls closer to the nuclear disc/ring and hence reaches larger "forbidden" velocities before crashing into the dustlane shock. This is a typical example of asymmetry 

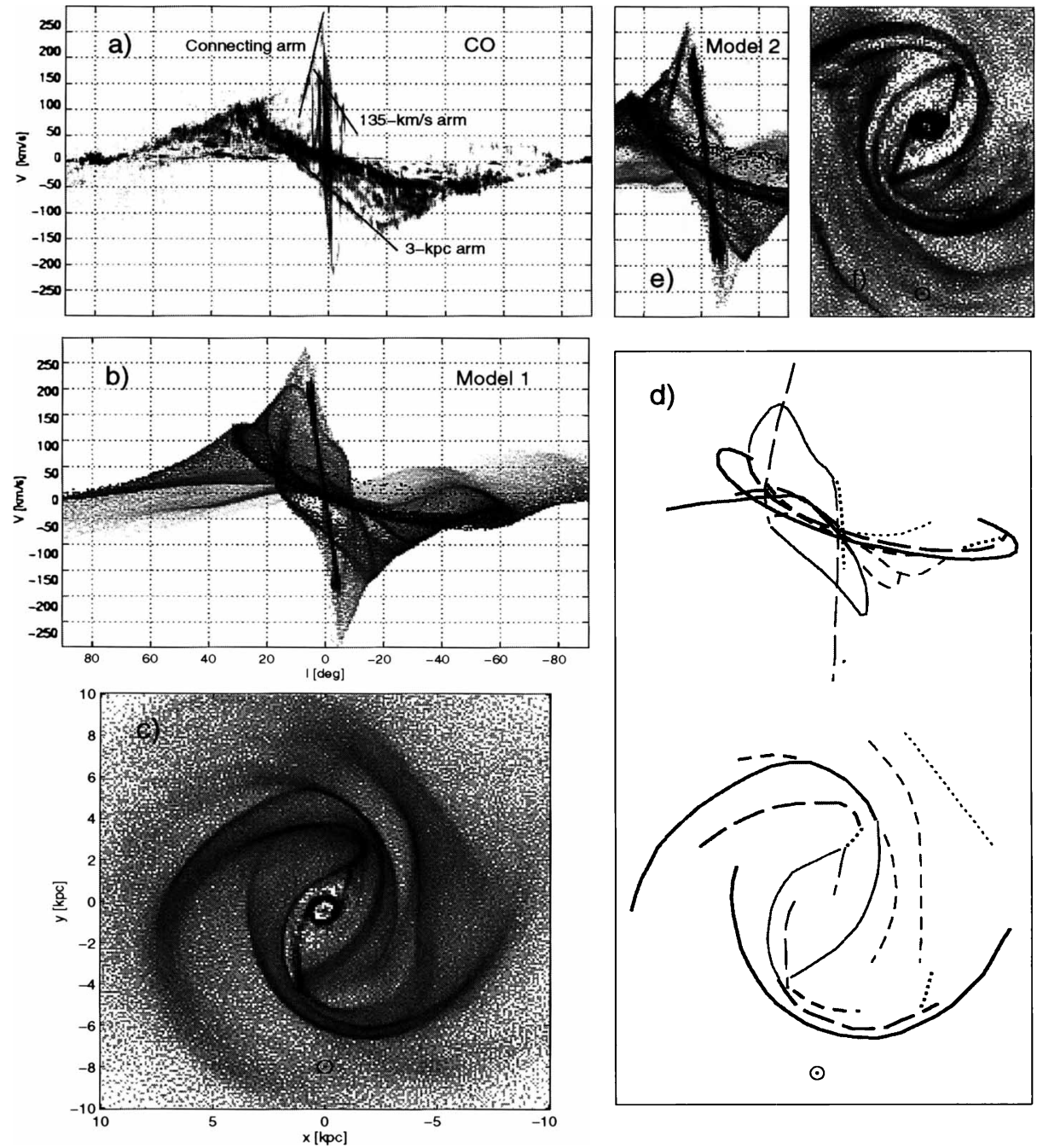

Figure 1. a) Observed CO $\ell-V$ diagram (Dame et al. 1995), with some relevant features indicated. b-c) Corresponding plot and face-on view for model 1 , which provides a fair global qualitative agreement with the data. d) Link between the spiral arms and the $\ell-V$ features in this model. e-f) Results for model 2, which reproduces almost perfectly the connecting arm. The location of the Sun is indicated by the $\odot$ symbol.

missed by gas flow calculations which do not take into account odd modes. Note also how well the models are able to reproduce the molecular ring.

\section{References}

Athanassoula E. 1992, MNRAS 259, 345

Dame et al. 1995, NCSA Astronomy Digital Image Library

Fux R. 1997, A\&A in press (astro-ph/9706242) 